\title{
Automatic Classification of Anterior Chamber Angle Based on Ultrasound Biomicroscopy Images
}

\author{
Jian Yu ${ }^{a, b, c}$ Wanyue Li ${ }^{d}$ Qian Chen ${ }^{a, b, c}$ Guohua Deng ${ }^{e}$ Chunhui Jiang ${ }^{a, b, c}$ \\ Guangxing Liu ${ }^{d}$ Guohua Shi $^{d}$ Xinghuai Sun ${ }^{a, b}, c$ \\ ${ }^{a}$ Department of Ophthalmology and Vision Science, Eye and ENT Hospital, Fudan University, Shanghai,

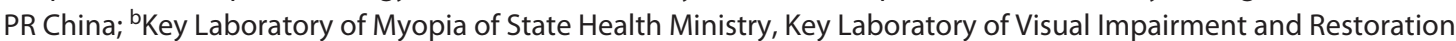 \\ of Shanghai, Shanghai, PR China; 'NHC Key Laboratory of Myopia, Fudan University, Shanghai, PR China; ${ }^{\text {dKey }}$

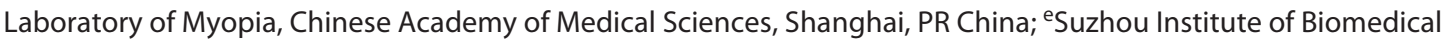 \\ Engineering and Technology, Chinese Academy of Sciences, Shanghai, PR China
}

\section{Keywords}

Ultrasound biomicroscopy · Anterior chamber angle ·

Automatic classification

\begin{abstract}
Introduction: Evaluating the anterior chamber angle (ACA) is important for the early diagnosis and treatment of primary angle-closure glaucoma. The assessment of ultrasound biomicroscopy (UBM) images usually requires well-trained ophthalmologists and screening for patients with narrow ACA is usually time- and labor-intensive. Therefore, the automatic assessment of UBM could be cost-effective and valuable in daily practice. Objective: The objective of this study is to develop an automatic method for localizing and classifying ACA based on UBM images. Methods: UBM images were collected and a coarse-to-fine method was used to localize the apex of the angle recess. By analyzing the grayscale features around the angle recess, closed angles were identified, and the rest were then classified as open or narrow angles, based on the degree of ACA. Using manual classification as the reference standard, the overall accuracy (OAcc), sensitivity (Sen), specificity (Spe), and balanced accuracy of the automatic classification method were evaluated. Results: A total
\end{abstract}

of 540 UBM images from 290 participants were analyzed. Using these UBM images and the proposed method, the ACA was classified as open, narrow, or closed. During processing, the method localized the angle recess with 95\% accuracy. The OAcc of the ACA classification was $77.8 \%$, and the Spe and Sen of our method were 85.8 and $81.7 \%$ for angle closure; 88.9 and $75.6 \%$ for open angles; 91.9 and $76.1 \%$ for narrow angles, respectively. Conclusions: Our method of automatic angle localization and classification based on UBM images is feasible and reliable. The automatic classification of ACA provides a basis and reference for future studies.

(c) 2020 S. Karger AG, Basel

\section{Introduction}

Glaucoma is the foremost cause of irreversible blindness and is also the second leading cause of blindness worldwide [1]. Primary angle-closure glaucoma (PACG) is a major form of glaucoma in Asia [1,2], and the evaluation of the anterior chamber angle (ACA) is important

Jian Yu and Wanyue Li contributed equally to this study and share first authorship.
Correspondence to:

Chunhui Jiang, chhjiang70@163.com Guangxing Liu, liugx@ sibet.ac.cn 

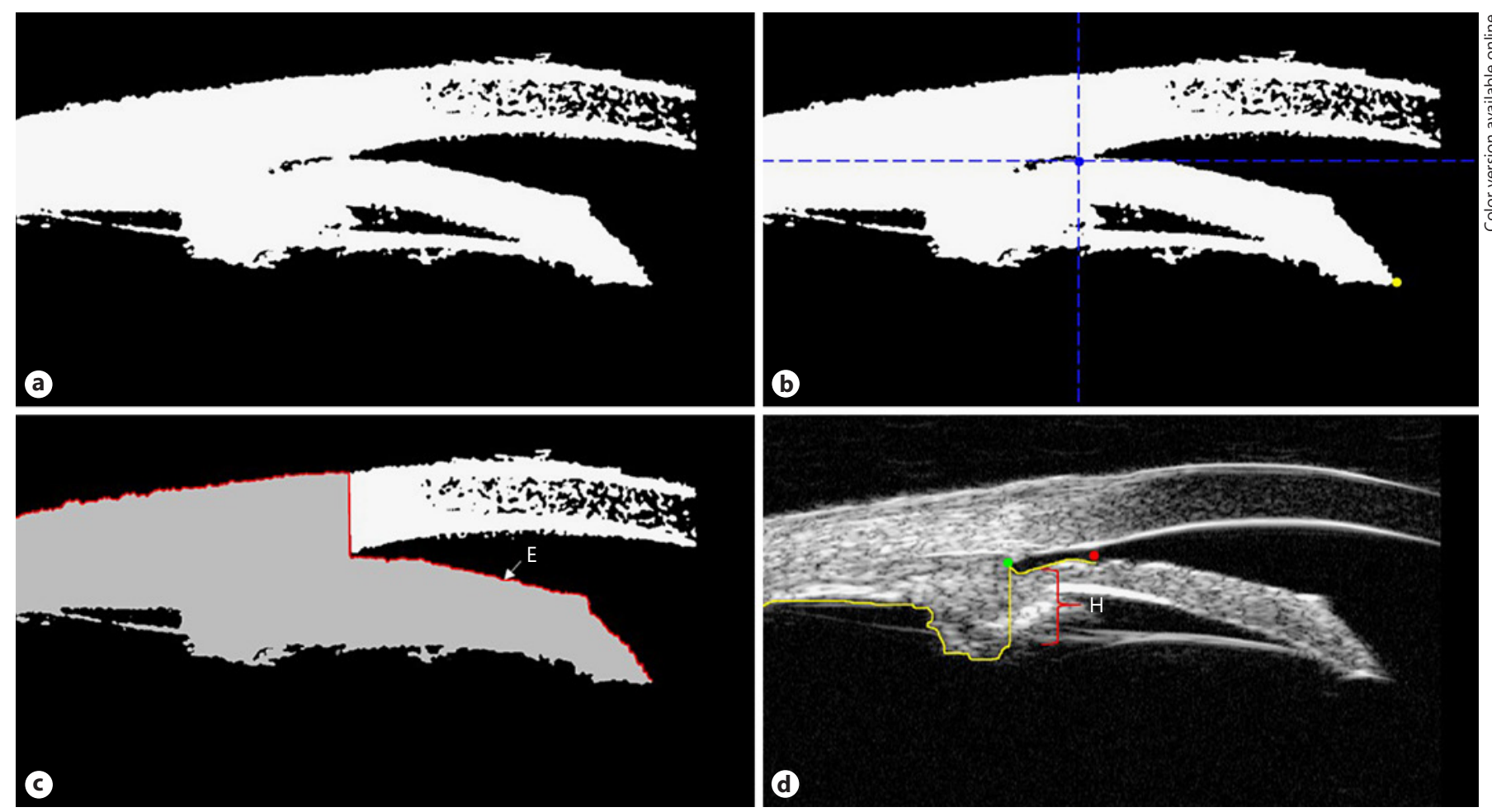

Fig. 1. Localization the apex of the iris recess. a Binary image with low threshold. b Localization of the center of gravity of the image (blue point) and the endpoint of the iris (yellow point). c Edge-searching curve of the iris. d Localization of the apex of the iris recess (coarse localization point is shown in red; fine localization point is shown in green).

for the early diagnosis and treatment of PACG. Many techniques, including slit-lamp microscopy [3], gonioscopy [4], anterior segment optical coherence tomography (AS-OCT) [5], and ultrasound biomicroscopy (UBM) [6], have been used for assessing ACA. UBM is particularly important because its resolution and penetration are good. However, the assessment of UBM images usually requires well-trained ophthalmologists, and screening for patients with narrow ACA who are at risk of developing angle-closure glaucoma (ACG), is usually time- and labor-intensive. Therefore, the automatic assessment of UBM could be cost-effective and valuable in daily practice. In this study, we propose an automatic method for localizing and classifying ACA based on UBM images that are expected to improve the screening and management of ACG.

\section{Methods}

In manual grading, the scleral spur is first identified, and then, the trabecular-iris angle (TIA) is measured. TIA is defined as the angle with its apex in the iris recess and the arms of the angle pass- ing through a point on the trabecular meshwork $750 \mu \mathrm{m}$ from the scleral spur and the point on the iris perpendicularly opposite [7]. The TIA was measured manually by 2 specialists and showed good consistency. The intraclass correlation coefficients (ICCs) between the 2 observers' manual measurements of total TIA, open angles, and narrow angles were $0.934,0.917$, and 0.911 , respectively. The average values were used for the subsequent study. Images with TIA $<15^{\circ}$ were classified as narrow angle, whereas those with TIA $\geq 15^{\circ}$ were classified as open angle. Images that showed contact between the trabecular meshwork and the peripheral iris were classified as closed angle.

The method of automatic processing is described in detail below. In brief, it followed the method used by human experts [8]. The angle apex in the iris recess was first localized with the intensity features of the iris root attachment points to identify the angle closure. ACA was measured and the rest of the images were classified as open or narrow angle. The proposed method aims to process UBM images like that shown in Figure 1, where ACA is facing directly right. Because it is difficult to obtain UBM images that are always in this form, a preprocessing operation, such as rotating or flipping, is performed manually to transform the original image to a specific format before the UBM image is processed. The image transformation rule is that the ACA of the transformed image should be facing right, and the angle bisector of the ACA should be nearly horizontal. 


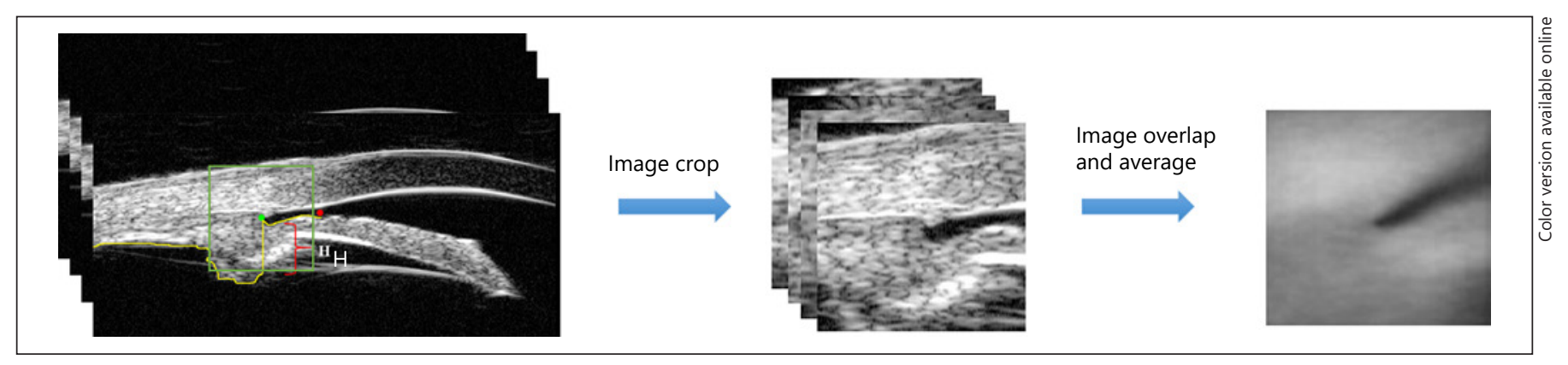

Fig. 2. Steps in obtaining the average image around the ACA area.

\section{Datasets}

To test the algorithm, a total of 540 UBM images from 290 participants (see online suppl. Table 1; for all online suppl. material, see www.karger.com/doi/10.1159/000510924) were collected between January and December 2017, at the Eye and ENT Hospital of Fudan University, Shanghai, China. UBM (MD-300L, Meda Co., Ltd., Tianjin, China; $50-\mathrm{MHz}$ probe transducer) examinations were performed by experienced doctors under normal room lighting with the patients lying on a bed in a supine position. Tetracaine (1\%) was applied to induce topical anesthesia. An eyecup with a diameter of 18-24 mm was placed in the conjunctival sac according to the size of the eye and was then filled with care solution or normal saline. Central scans in the vertical and horizontal axes were taken, together with scans at the following 8 positions: 12:00, $1: 30,3: 00,4: 30,6: 00,7: 30,9: 00$, and 10:30. If both eyes undertook UBM test, then both eyes were included. Eyes with history of intraocular surgery were excluded from the study. One representative image for each eye was selected for the study.

\section{Localizing the Apex of the Angle Recess}

ACA localization is essential for localizing the ACA recess accurately. A manual labeling method [9] or an automatic method of edge detection [10] is often used. However, the edge detection method is sensitive to the image quality, and UBM images do not always have sharp contrast and a high signal-to-noise ratio, which limits the use of this method. Therefore, we used a coarse-to-fine method to localize the apex of the angle recess.

\section{Coarse Localization of the Apex of the Angle Recess}

To preserve more details of the ACA, a low threshold was initially used for image binarization (Fig. 1a). Next, the image was separated into 4 parts according to the center of gravity of the image (Fig. 1b). The endpoint of the iris was determined by segmenting the area in the bottom right of the image, using the endpoint of the iris as the starting point from which to fit the upper edge of the iris. During the search for the edge points of the iris, the height and position of the edge point changed suddenly, and this jump point was identified as the coarse apex of the iris recess $\left(x_{\text {suspected }}, y_{\text {suspected }}\right)$. In Figure 1c, the edge-searching curve is labeled $E$ and shown in red, and the red point in Figure 1d is the coarse localization point.

Fine Localization of the Apex of the Angle Recess

The fine localization of the apex of the angle recess was determined in the original UBM image. Because the coarse localization of the apex of the angle recess was performed with the binary im- age, which had a low threshold, the actual apex was to the left of the coarse apex. For each column, from line $x=x_{\text {suspected }}$ leftward, and from line $y=y_{\text {suspected }}$ downward, the difference in the grayscale value for each point and for the coarse localization point was calculated. If the difference was less than 30 pixels, the grayscale value of the point was similar to that of the coarse localization point, and the locations of similar grayscale points were all recorded. When the vertical coordinate of similar grayscale points jumped on both sides of the angle recess, the jump point was determined, and the apex of the angle recess $\left(x_{\text {point }}, y_{\text {point }}\right)$ was localized. The height of the jump was the thickness of iris at the root (marked " $H$ " in Fig. 1d). The green point shown in Figure $1 \mathrm{~d}$ is the fine localization point. In summary, finding the fine localization point depends on 2 conditions: (i) the gray scale of this point is similar to the coarse localization point and (ii) this point jumps on both sides of the angle recess.

The apex of the angle recess is defined as the point at which the fitting curve of the inner iris intersects that of the inner cornea [7]. If the fine localization point coincided with this point, the apex of the angle recess was located successfully. The accuracy of the angle recess localization was defined as the ratio of the number of successful fine-localized images to the number of total images.

\section{Basis of ACA Classification}

First, by analyzing the grayscale features around the angle recess, the closed angles are identified, and the rest are then classified as open or narrow, based on the degree of ACA.

\section{Analysis of the Grayscale Features of the Angle Recess}

To analyze the grayscale features around the angle recess better, we computed the averaged images for the different types of angles. The steps used to average the images are shown in Figure 2. First, we selected and cropped a square region around the angle recess, in which the apex of the angle recess was the center of the square region and the thickness of the iris was half the length of the side of the square. The averaged image was then obtained by overlapping and averaging these cropped images.

The averaged images were each obtained by averaging $100 \mathrm{im}$ ages (Fig. 3). The grayscale value of the cornea just above the angle recess was higher than that in the corresponding region of the iris in the open-angle and narrow-angle cases (Fig. 3; Table 1). However, the grayscale value of the cornea above the angle recess in the angleclosure cases was lower than that of the iris. The ACA image was divided into the upper and lower parts according to the position of the apex of the angle recess, in which the upper part was the cornea 
Fig. 3. Average images of different angles. Angle closure (a); narrow angle (b); open angle (c).

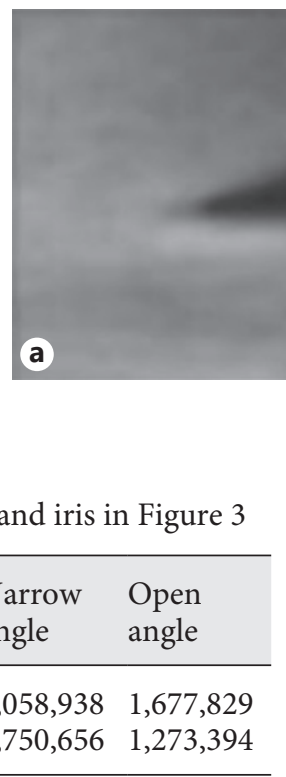

and the lower part was the iris. The location of the ACA region boundary was determined by the thickness of the iris. For iris thickness $H$, the region near the iris insertion point was determined by taking $\left(y_{\text {point }}-H, y_{\text {point }}+H\right)$ in the vertical direction and $\left(x_{\text {point }}-H\right.$, $\left.x_{\text {point }}+H\right)$ in the horizontal direction, the image was divided into the cornea (upper) and iris (lower) regions based on the line $y=$ $y_{\text {point }}$. The closed angle was identified by comparing the differences in the grayscale values between the cornea and iris regions.

\section{Estimating the ACA Angle}

The iris and cornea around the ACA were determined according to the localization of the apex of the angle recess and the boundary of the anterior surface of the iris determined in Datasets section (Fig. 4a, b). A diagram of the process used to determine TIA is shown in Figure 5 [7]: point $s$ is the apex of the angle recess, with $s$ as the center of the circle with a radius of $750 \mu \mathrm{m}$; the 2 points at which the circle intersects the inner surface of the cornea and the upper surface of the iris are found, and then, the TIA is determined. If the ACA is $\geq 15^{\circ}$ [11], it is classified as open; otherwise, it is classified as narrow.

\section{Statistics}

Using manual classification as the reference standard, the overall accuracy (OAcc), sensitivity (Sen), specificity (Spe), and balanced accuracy (BAcc) of the automatic classification method were calculated. Sen is the proportion of positive samples that are correctly predicted as positive. Spe is the proportion of negative samples that are correctly predicted as negative. BAcc is used to evaluate the performance of the classifier on unbalanced data. The OAcc, Sen, Spe, and BAcc are expressed as:

$$
\begin{aligned}
& \mathrm{OAcc}=\frac{\mathrm{TP}+\mathrm{TN}}{\mathrm{TP}+\mathrm{FP}+\mathrm{FN}+\mathrm{TN}} \\
& \mathrm{Sen}=\frac{\mathrm{TP}}{\mathrm{TP}+\mathrm{FN}}
\end{aligned}
$$

(b)

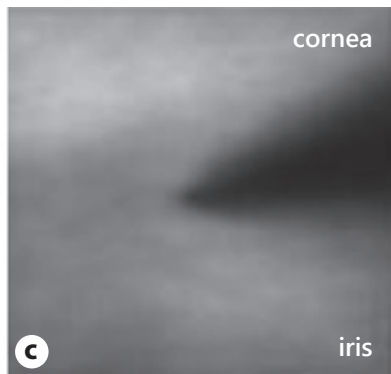

$$
\mathrm{Spe}=\frac{\mathrm{TN}}{\mathrm{TN}+\mathrm{FP}}
$$

$$
\mathrm{BAcc}=\frac{1}{2}(\text { sensitivity }+ \text { specificity })
$$

where TP is true positives, defined as the number of cases correctly classified as angle closure; TN is true negatives, defined as the number of cases correctly classified as another angle type; FP is false positives, defined as the number of cases incorrectly classified as angle closure; and FN is false negatives, defined as the number of cases incorrectly classified.

To evaluate the accuracy of TIA measurements with our method, the mean absolute percentage error was used, which was calculated as

$$
\text { MAPE }=\frac{1}{N} \sum_{j-1}^{N}\left|\frac{\alpha_{j}-\hat{\alpha}_{j}}{\alpha_{j}}\right|
$$

where $\alpha$ is a manually measured value and $\hat{\alpha}$ is the value measured with our method.

In this study, the manual and the automatic methods are treated statistically as 2 observers. To evaluate the consistency between the manually measured values and the values measured with our method, the measurements obtained independently from the 2 observers were used to calculate the coefficient of variation (the standard deviation [SD] of the differences divided by the overall mean) and the ICC. $p<0.05$ was considered statistically significant.

\section{Results}

\section{Accuracy of ACA Localization}

The average accuracy of angle recess localization was 95\%; 97.2\% for open angles, $96.1 \%$ for closed angles, and $91.7 \%$ for narrow angles (Table 2 ).

\section{Angle-Closure Classification}

The 540 images in our dataset were classified as closed angle or not. The BAcc was 0.850, and the Spe and Sen were 0.883 and 0.817 , respectively. The confusion matrix is shown in Table 3. 

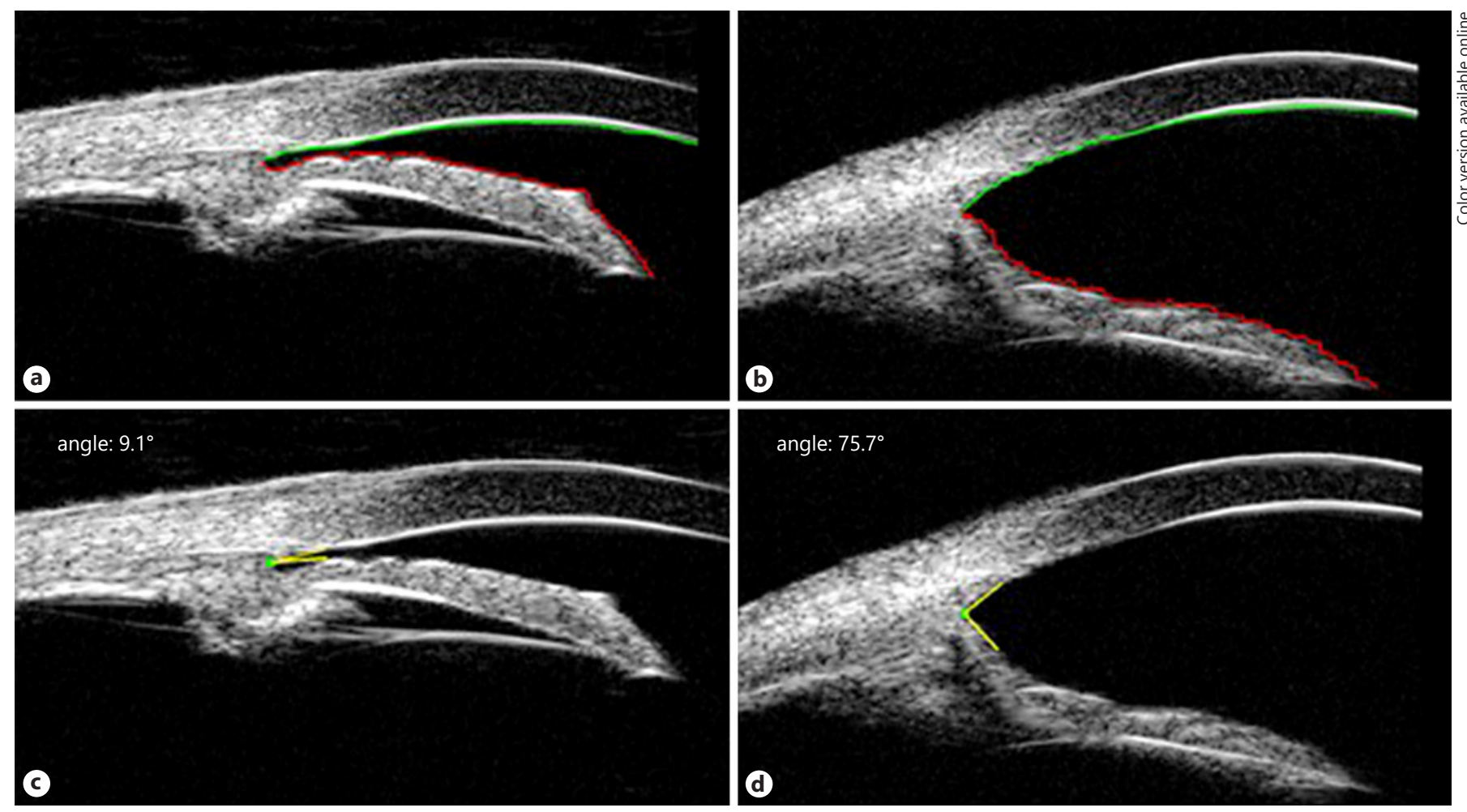

Fig. 4. Estimate of the degree of ACA. $\mathbf{a}, \mathbf{b}$ Fitting the upper edge of the iris and the lower edge of the cornea (green curve: lower edge of cornea; red curve: upper edge of iris). c Estimation of the ACA $\left(^{\circ}\right.$ ) of the narrow angle. $\mathbf{d}$ Estimation of the ACA $\left(^{\circ}\right)$ of the open angle.

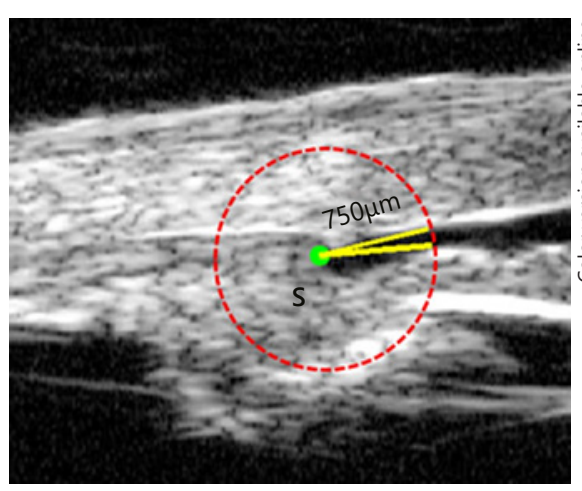

Fig. 5. Diagram of the process by which TIA is determined. Point $\mathrm{s}$ is the apex of angle recess, with $\mathrm{s}$ as the center of the circle with a radius of $750 \mu \mathrm{m}$; the 2 points at which the inner surface of the cornea and the upper surface of the iris intersect are identified, and TIA can then be determined.

\section{Evaluation of TIA Measurements}

Using the manual results as the standard, the ICCs between the manual and automatic measurements of total TIA, open angles, and narrow angles were $0.83,0.88$, and 0.63 , respectively (Table 4 ).
Table 2. Accuracy of ACA localization

\begin{tabular}{|c|c|c|c|c|}
\hline & Images & $\begin{array}{l}\text { Successful } \\
\text { coarse } \\
\text { localization } \\
\text { images }\end{array}$ & $\begin{array}{l}\text { Successful fine } \\
\text { localization } \\
\text { images }\end{array}$ & $\begin{array}{l}\text { Total } \\
\text { accuracy, } \\
\%\end{array}$ \\
\hline Open angle & 180 & 180 & 175 & 97.2 \\
\hline Angle closure & 180 & 176 & 173 & 96.1 \\
\hline Narrow angle & 180 & 178 & 165 & 91.7 \\
\hline
\end{tabular}

\section{Evaluation of ACA Classification}

The 540 images were classified as open-angle, narrow-angle, or angle-closure images. The OAcc of the ACA classification was $77.8 \%$. The Spe and Sen of our method were 85.8 and $81.7 \%$, respectively, for angle closure; 88.9 and $75.6 \%$, respectively, for open angles; and 91.9 and $76.1 \%$, respectively, for narrow angles (Table 5). 
Table 3. Confusion matrix of angle-closure classification

\begin{tabular}{lll}
\hline & $\begin{array}{l}\text { Predicted as } \\
\text { angle closure }\end{array}$ & $\begin{array}{l}\text { Predicted as } \\
\text { another angle }\end{array}$ \\
\hline $\begin{array}{l}\text { Angle closure } \\
\text { Another angle }\end{array}$ & $\mathrm{TP}=147$ & $\mathrm{FN}=33$ \\
Spe & $\mathrm{FP}=42$ & $\mathrm{TN}=318$ \\
Sen & 0.883 & \\
BAcc & 0.817 & \\
\hline
\end{tabular}

$\mathrm{TP}$, true positives; TN, true negatives; FP, false positives; FN, false negatives; Sen, sensitivity; Spe, specificity; BAcc, balanced accuracy.

Table 5. Automated ACA classification compared with manual classification

\begin{tabular}{lllll}
\hline $\begin{array}{l}\text { Manual } \\
\text { classification }\end{array}$ & $N$ & \multicolumn{2}{l}{ Automated ACA classification } \\
\cline { 3 - 5 } & & $\begin{array}{l}\text { angle } \\
\text { closure }\end{array}$ & $\begin{array}{l}\text { open } \\
\text { angle }\end{array}$ & $\begin{array}{l}\text { narrow } \\
\text { angle }\end{array}$ \\
\hline Angle closure & 180 & 147 & 21 & 12 \\
Open angle & 180 & 27 & 136 & 17 \\
Narrow angle & 180 & 24 & 19 & 137 \\
\hline Spe & & 0.858 & 0.889 & 0.919 \\
Sen & & 0.817 & 0.756 & 0.761 \\
Accuracy & & 0.817 & 0.756 & 0.761 \\
\hline
\end{tabular}

OAcc $\quad 0.778$

ACA, anterior chamber angle; OAcc, overall accuracy; Sen, sensitivity; Spe, specificity.

\section{Discussion}

Using UBM images and our method, the angle recess was localized with 95\% accuracy, and the ACA was classified as open, narrow, or closed. The first attempt to grade the ACA was by Gradle and Sugar [12], after which Scheie [13] and Shaffer [14] proposed their grading systems, which have been widely used. Recently, automatic image processing has been widely investigated but reports of the automatic localization and classification of the ACA are still limited. The Zhongshan Angle Assessment Program provides a semiautomatic algorithm for calculating various parameters of the ACA using images acquired by AS-OCT (Visante AS-OCT; Carl Zeiss Meditec, Dublin, CA, USA), but this method requires the manual determination of the scleral spur [15]. In other studies [8, 16], AS-OCT images were divided into open and closed ACA categories, but the classification of nar-

Automatic Classification of Anterior Chamber Angle by UBM
Table 4. Evaluation of TIA measurements

\begin{tabular}{llll}
\hline & $\begin{array}{l}\text { Accuracy } \\
\text { (MAPE) }\end{array}$ & CV (\%) (95\% CI) & ICC (95\% CI) \\
\hline Total angle & 0.714 & $17.5(8.7-26.3)$ & $0.83(0.79-0.86)$ \\
Open angle & 0.827 & $9.9(7.6-12.2)$ & $0.88(0.78-0.93)$ \\
Narrow angle & 0.601 & $25.1(15.6-34.6)$ & $0.63(0.28-0.78)$ \\
\hline
\end{tabular}

TIA, trabecular-iris angle; MAPE, mean absolute percentage error; $\mathrm{CV}$, coefficient of variation; ICC, intraclass correlation coefficient; CI, confidence intervals.

row angles was not included. In this paper, we present an algorithm for the automatic localization and classification of ACA that differs from earlier methods in that it was developed to process UBM images. Compared with AS-OCT, in vivo UBM can penetrate dense tissues (e.g., the sclera, iris, and ciliary body), and thus provides results that are more detailed $[17,18]$, which are invaluable in the diagnosis and management of glaucoma, especially PACG. OCT is also a clearer imaging technology than UBM $[18,19]$, and our method should be applicable to OCT images and may also have a wider range of applications.

In the manual part of the analysis, we defined TIA $<15^{\circ}$ as a narrow angle and TIA $\geq 15^{\circ}$ as an open angle. Although there is no universally accepted standard for the classification of open and narrow angles in UBM, in a population-based study of UBM in Japan [20], the average TIA was $10.3 \pm 3.9^{\circ}$ in patients with PACG or suspected PACG based on gonioscopic findings, whereas it was $24.2 \pm 9.3^{\circ}$ in the healthy control group. Therefore, in the present study, we used $15^{\circ}$ as the cutoff for the boundary between narrow- and open-angle cases because this value is about $1 \mathrm{SD}$ above the average value for suspected PACG or PACG $\left(10.3^{\circ}+3.9^{\circ}=14.2^{\circ}\right)$ and 1 SD below the average value for normal participants $\left(24.2^{\circ}-\right.$ $9.3^{\circ}=14.9^{\circ}$ ).

In our method, the algorithm first determines the apex of the ACA, and then the TIA is measured and the ACA is classified according to this information. Therefore, this method is highly dependent on the accuracy of the apex localization. In this study, we proposed a coarseto-fine method to localize the apex of the angle recess. The coarse localization point is determined by finding the point that jumps on both sides of the angle recess during the fitting of the inner iris on the binary image. The fine localization point is determined by finding the point that has similar grayscale characteristics to those of the coarse point and jumps on both sides of the angle recess. 
Table 6. Comparison of present method with other methods

\begin{tabular}{llccc}
\hline & Imaging manners & BAcc & Spe & Sen \\
\hline Method (Xu et al. [16]) & Visante AS-OCT & 0.758 & 0.850 & 0.666 \\
Method (Xu et al. [8]) & Visante AS-OCT & 0.842 & 0.850 & 0.834 \\
Method (Fu et al. [7]) & Visante AS-OCT & 0.872 & 0.862 & 0.886 \\
Method(Fu et al. [7]) & Cirrus HD-OCT & 0.802 & 0.850 & 0.753 \\
Method (Ni et al. [21]) & Casia SS-1000 OCT & 0.991 & 0.961 & 0.999 \\
Our method & UBM & 0.850 & 0.883 & 0.817 \\
\hline
\end{tabular}

BAcc, balanced accuracy; Spe, specificity; Sen, sensitivity; UBM, ultrasound biomicroscopy; OCT, optical coherence tomography.

This algorithm localizes the angle recess with an accuracy of $95 \%$.

Many other authors have classified the ACA as closed and nonclosed [7, 8, 16, 21]. For ease of comparison, we also classified the images into 2 types. Table 6 shows that the BAcc, Spe, and Sen of our methods are similar to those of the 3 other reported methods. Ni et al. [21], using a classification algorithm based on fuzzy clustering, achieved good closed angle classification, but the algorithm could only be applied to high-resolution images acquired with a CASIA OCT system (SS-1000, Tomey).

To classify open and narrow angles, TIA was measured automatically. The evaluation of the TIA measurement is shown in Table 4. Compared with manual measurement, the ICC was good for open angles (0.88), but poor for narrow angles (0.63). The accuracy of the TIA measurement in this study was low (only about 0.63 for narrow angles). However, because we used TIA $=15^{\circ}$ as the threshold to define whether ACA was open or narrow, the precision of the TIA measurement was not very important, whereas the decision whether TIA was $\geq 15^{\circ}$ was important. As illustrated in online suppl. Table 2 , the proposed method of that decision had an accuracy of 0.884 and thus could discriminate open and narrow angles.

The accuracy of apex localization was higher for open angles and closed angles than for narrow angles (Table 2), mainly because the area of a narrow angle between the iris and the cornea is small. Moreover, in some cases, the bulge of the iris root causes ACA to form an isolated closed area.

Unlike a recently reported algorithm based on deep learning [6], our method is based on image processing. Although the accuracy of our method is not as high as that of deep-learning-based methods, the method described here provides more details of the processing, especially localizing the apex of the angle recess and the edge of the ACA. This could be an advantage over the black-box deep-learning technique. By monitoring these steps during processing, aberrant steps or misgraded ones can be identified and corrected. The combination of these 2 methods might provide an automatic way to achieve high accuracy and, at the same time, monitor the individual steps of the process.

EA large proportion of PACG patients is unaware of their condition until their first acute attack [22]. UBM and the method described here could be used to identify patients at high risk of developing acute PACG, and the suffering of these patients could be reduced or even avoided with phacoemulsification or prophylactic laser peripheral iridotomy, which can increase the median angle width [23]. This method could also be used as a remote model, which would be especially useful for people in rural areas, who have a high incidence of PACG [24] but limited medical resources.

Only Chinese subjects and images acquired by one UBM system were included in this study, so the performance of the method in processing images captured by other UBM systems or images from subjects from other ethnic backgrounds requires further investigation. Moreover, the results of gonioscopy were not presented here, so a comparison of this image processing method and gonioscopy is still necessary. Whether a combination of image processing and artificial intelligence could improve the accuracy of the results should also be examined.

In summary, we developed an algorithm for the automatic classification of ACA based on UBM images. We confirmed experimentally that our automatic method of angle localization and classification is feasible and reliable. The automatic classification of ACA provides a basis and reference for future work. 


\section{Statement of Ethics}

The study was approved by the Ethics Committee of the Eye and ENT Hospital of Fudan University (No. ky2012-037) and adhered to the Declaration of Helsinki, written informed consent was obtained from all patients.

\section{Conflict of Interest Statement}

The authors have no proprietary or commercial interests in any of the materials discussed in this article and no other conflicts of interest to declare.

\section{Funding Sources}

Publication of this article was supported, in part, by research grants from the National Key Research \& Development Plan (2017YFC0108200, 2017YFC0108201), the Shanghai Committee of Science and Technology (19441900900), and the Jiangsu Province Key Research and Development Program (BE2018667).

\section{Author Contributions}

J.Y., W.L., C.J., and G.L. contributions to design and conduct the work, Q.C., G.D., G.S., and X.S. contributions to revise the manuscript.

\section{References}

1 Quigley HA, Broman AT. The number of people with glaucoma worldwide in 2010 and 2020. Br J Ophthalmol. 2006;90(3):262-7.

2 Foster PJ, Johnson GJ. Glaucoma in China: how big is the problem? Br J Ophthalmol. 2001;85(11):1277-82.

3 Dinc UA, Oncel B, Gorgun E, Yalvac IS. Assessment of anterior chamber angle using Visante OCT, slit-lamp OCT, and Pentacam. EUR J Ophthalmol. 2010;20(3):531-7.

4 Phu J, Wang H, Khuu SK, Zangerl B, Hennessy MP, Masselos K, et al. Anterior chamber angle evaluation using gonioscopy: consistency and agreement between optometrists and ophthalmologists. Optom Vis Sci. 2019; 96(10):751-60

5 Pinilla LI, Lopez DLFC, Segura F, Orduna HE Sanchez-Cano A. Evaluation of anterior chamber parameters with spectral-domain optical coherence tomography. Jpn J Ophthalmol. 2018;62(2):209-15.

6 Shi G, Jiang Z, Deng G, Liu G, Zong Y, Jiang $\mathrm{C}$, et al. Automatic classification of anterior chamber angle using ultrasound biomicroscopy and deep learning. Transl Vis Sci Technol. 2019;8(4):25

7 Fu H, Xu Y, Lin S, Zhang X, Wong DWK, Liu J, et al. Segmentation and quantification for angle-closure glaucoma assessment in anterior segment OCT. IEEE Trans Med Imaging. 2017;36(9):1930-8.

8 Xu Y, Liu J, Cheng J, Lee BH, Wong DW, Baskaran M, et al. Automated anterior chamber angle localization and glaucoma type classification in OCT images. Conf Proc IEEE Eng Med Biol Soc. 2013;2013:7380-3.
9 Leung CK, Li H, Weinreb RN, Liu J, Cheung CY, Lai RY, et al. Anterior chamber angle measurement with anterior segment optical coherence tomography: a comparison between slit lamp OCT and Visante OCT. Invest Ophthalmol Vis Sci. 2008;49(8):3469-74.

10 Leung CK, Yung WH, Yiu CK, Lam SW, Leung DY, Tse RK, et al. Novel approach for anterior chamber angle analysis: anterior chamber angle detection with edge measurement and identification algorithm (ACADEMIA). Arch Ophthalmol. 2006;124(10): 1395-401.

11 Mannino G, Abdolrahimzadeh B, Calafiore S, Anselmi G, Mannino C, Lambiase A. A review of the role of ultrasound biomicroscopy in glaucoma associated with rare diseases of the anterior segment. Clin Ophthalmol. 2016;10: 1453-9.

12 Gradle HS, Sugar HS. Concerning the chamber angle. Am J Ophthalmol. 1940;23(10): $1135-9$.

13 Scheie HG. Width and pigmentation of the angle of the anterior chamber; a system of grading by gonioscopy. AMA Arch Ophthalmol. 1957;58(4):510-2.

14 Shaffer RN. Primary glaucomas. Gonioscopy, ophthalmoscopy and perimetry. Trans Am Acad Ophthalmol Otolaryngol. 1960;64:11227.

15 Console JW, Sakata LM, Aung T, Friedman DS, He M. Quantitative analysis of anterior segment optical coherence tomography images: the Zhongshan Angle Assessment Program. Br J Ophthalmol. 2008;92(12):1612-6.

16 Xu Y, Liu J, Tan NM, Lee BH, Wong DW, Baskaran M, et al. Anterior chamber angle classification using multiscale histograms of oriented gradients for glaucoma subtype identification. Conf Proc IEEE Eng Med Biol Soc. 2012;2012:3167-70.
17 Pavlin CJ, Harasiewicz K, Sherar MD, Foster FS. Clinical use of ultrasound biomicroscopy. Ophthalmology. 1991;98(3):287-95.

18 Nolan W. Anterior segment imaging: ultrasound biomicroscopy and anterior segment optical coherence tomography. Curr Opin Ophthalmol. 2008;19(2):115-21.

19 Wang D, Pekmezci M, Basham RP, He M, Seider MI, Lin SC. Comparison of different modes in optical coherence tomography and ultrasound biomicroscopy in anterior chamber angle assessment. J Glaucoma. 2009; 18(6):472-8.

20 Henzan IM, Tomidokoro A, Uejo C, Sakai H, Sawaguchi S, Iwase A, et al. Comparison of ultrasound biomicroscopic configurations among primary angle closure, its suspects, and nonoccludable angles: the Kumejima Study. Am J Ophthalmol. 2011;151(6):106573.e1.

21 Ni NS, Tian J, Marziliano P, Wong HT. Anterior chamber angle shape analysis and classification of glaucoma in SS-OCT images. Ophthalmol. 2014;2014:942367.

22 Sun X, Dai Y, Chen Y, Yu DY, Cringle SJ, Chen J, et al. Primary angle closure glaucoma: what we know and what we don't know. Prog Retin Eye Res. 2017;57:26-45.

23 Zebardast N, Kavitha S, Krishnamurthy P, Friedman DS, Nongpiur ME, Aung T, et al. Changes in anterior segment morphology and predictors of angle widening after laser iridotomy in South Indian eyes. Ophthalmology. 2016;123(12):2519-26.

24 Song P, Wang J, Bucan K, Theodoratou E, Rudan I, Chan KY. National and subnational prevalence and burden of glaucoma in China: a systematic analysis. J Glob Health. 2017; 7(2):20705. 